\title{
Application of fast pressure sensitive paint in hypervelocity flow
}

\author{
W. Flaherty, J. Crafton† G. S. Elliott $\ddagger$ and J. M. Austin ${ }^{\S}$
}

\begin{abstract}
The development of fast responding pressure sensitive paints for measurements in highenthalpy, hypersonic flows is reported. Data are obtained for flat plate boundary layers in an impulse facility with typical test gas times on the order of hundreds of microseconds. The fast pressure sensitive paint is found to have the required response time and sufficient signal level to visualize vortical boundary layer structures in a Mach 5.12 freestream. The flow behind protuberance strips with different geometries is evaluated with measurements of the vortex frequency and diameter, as well as breakdown distance. Comparisons with static pressure measurements made using pressure taps show differences of up to a factor of three, and calibration sources as well as an alternative lifetime measurement method remain to be investigated.
\end{abstract}

\section{Introduction}

Fluorescent paints which are sensitive to pressure or temperature can provide full-field data over a threedimensional surface, a significant advantage over point measurements. While value of these paints has been demonstrated in numerous applications, the relatively slow response time has precluded their use in many hypersonic impulse facilities. Traditionally, even "fast" pressure sensitive paints (PSPs) had response times in the range of milliseconds while test times in impulse facilities can be on the order of hundreds of microseconds. In addition, the experimental conditions in impulse facilities are typically very challenging with high shear and heat transfer rates, and survivability issues due to dynamic loads and particulate damage are encountered. In this study, a PtTFPP-based porous polymer paint was used to achieve response times on the order of 30-50 $\mu \mathrm{s}$. This paper demonstrates the use of these paints for surface measurements in high enthalpy, hypersonic flows created in an impulse facility with test times on the order of 100-400 $\mu$ s.

Pressure sensitive paints operate through the oxygen quenching of a luminescent molecule. When a luminescent molecule absorbs a photon, it transitions to an excited singlet energy state. The molecule then typically recovers to the ground state by the emission of a photon of longer wavelength. In some materials oxygen can interact with the molecule such that the transition to the ground state is non-radiative. The rate at which these two processes compete is dependent on the partial pressure of oxygen, and thus the output intensity of the paint (assuming a uniform light source) is proportional to the static pressure of the air test gas. The paint is applied to the surface of the model, and conventional imaging techniques are used to record the intensity distribution during the test time. A long-pass filter is used to isolate the paint luminescence from the illumination. One issue with PSP is that the luminescence of the paint is not only a function of pressure. Factors such as paint thickness, uneven illumination, concentration of the luminescent molecule, and non-uniformities on the imaging CCD can all result in fluctuations in the recorded intensity. To minimize these effects, a ratio is taken between the image taken during the test time and an image taken with no flow over the model at a known pressure. These are designated the "wind-on" and "wind-off" images respectively.

Typical paint formulations are a mixture of the luminescent molecule and a polymer binder which is used to create the bond between the luminescent molecule and the model. The response times are dependent on the rate at which the oxygen molecules can permeate the binder before they can quench the luminescent

\footnotetext{
*Graduate student, University of Illinois, Urbana IL 61801

${ }^{\dagger}$ Research Scientist, Innovative Scientific Solutions, Inc. Dayton OH, 45440, Senior Member AIAA

${ }^{\ddagger}$ Professor, University of Illinois, Urbana IL 61801, Senior Member AIAA

$\S$ Assistant Professor, University of Illinois, Urbana IL 61801, Senior Member AIAA
} 
molecules. Fast response times for typical polymer binder PSP are a few tenths of a second. When compared to the typical test times in impulse facilities capable of high velocity, high enthalpy flows such as expansion tubes (hundreds of microseconds) or reflected shock tunnels (a few milliseconds), it is obvious that these paints are grossly inadequate for capturing any of the flow structures.

The response time of a given PSP is a function of the diffusion coefficient of the polymer binder and the thickness of the paint layer. The relationship between response time $\tau_{d i f f}$, diffusion coefficient $D_{m}$, and paint thickness $h$ is typically given as:

$$
\tau_{\text {diff }} \sim \frac{h^{2}}{D_{m}}
$$

Obviously, decreasing the paint thickness will reduce the response time, but the signal intensity also decreases. Another method to increase the speed of oxygen permeation is the use of porous binders. The advantage of a porous binder is that the oxygen has much easier access to the luminescent molecules, allowing the quenching to occur much faster. In addition, the effective surface area of a porous binder is much larger than the non-porous counterpart, resulting in higher radiative intensity. One downside of these binders is that since the oxygen has such easy access to the luminescent molecules, too much quenching can result, so that at higher pressures the signal is low. It has been found that these paints are well suited for use in facilities where the static pressure is below around $20 \mathrm{kPa}$.

Development of "fast" PSP's with porous binders has been the subject of much research. The ability to create a paint with good signal-to-noise with microsecond response time would be extremely useful not only for short duration facilities like the HET, but also to unsteady flows whos characteristic time scales are on the order of microseconds. ${ }^{1}$ To date, three types of fast PSP have been developed: anodized aluminum (AA) PSP, thin-layer chromatography (TLC) PSP, and polymer/ceramic PSP. AA PSP was originally developed by Asai $^{2}$ for application to cryogenic wind tunnels. AA PSP is done by anodizing an aluminum model to create a consistent honeycomb structure across the surface of the model. After this is done the luminophore can be adsorbed onto the surface of the model to create the PSP. Asai's method was further improved by Sakaue and Sullivan. ${ }^{3}$ In this study, the response of AA PSP was compared to both TLC and polymer/ceramic PSP. They found that the best response time was given by the AA PSP, $34.8 \mu \mathrm{s}$. The TLC was on the same order as the AA $(65.1 \mu \mathrm{s})$, and the polymer/ceramic was around $345 \mu \mathrm{s}$. The number for the polymer/ceramic is actually much higher than what is attainable with this type, as the paint was not optimized. One benefit of the AA PSP is that the thickness can be directly controlled by varying the anodization time of the model. This allows for much more direct control over the response time of the gauge. Conversely, the authors of that study were unable to vary the thickness of the TLC PSP, which resulted in much less control over the paint. TLC PSP was first developed by Baron et al.. In this method, the luminophore is adsorbed onto the surface of a thin-layer chromatography sheet which can be purchased commercially. Baron et al. were able to get response times below $25 \mu \mathrm{s}$ with their TLC PSP, and it was theorized that response times as low as $10 \mu$ s could be achieved. The drawbacks to this type of PSP are that is can only be applied to a flat plate, it does not have a large dynamic range, and it is brittle compared to the other two types (making it a poor choice for use in impulse facilities).

Polymer/ceramic PSP was the type used in this current experimental study. It combines the properties of porous and tradiational PSPs to obtain fast response time while good signal at higher pressures is maintained. A coating containing luminescent molecules with a short lifetime is typically applied onto the polymer/ceramic paint. In previsous studies, these PSPs were demonstrated to measure pressure fluctuations of up to $20 \mathrm{kHz} .{ }^{4}$ For a much more in depth review of the theory and development of these fast response PSPs see Gregory, et al. ${ }^{4}$

\section{Experimental setup}

Experiments were carried out in the Hypervelocity Expansion Tube (HET) at the University of Illinois. ${ }^{5}$ The HET is $9.14 \mathrm{~m}$ long with an inner diameter of $150 \mathrm{~mm}$. A range of test conditions by changing the gas composition and pressures in the three different sections of the facility. The HET is currently capable of stagnation enthalpies from 4.5 to $8.0 \mathrm{MJ} / \mathrm{kg}$ and Mach numbers from 3.0 to 7.5. For the present study, a single test condition was used, Table 1. This test condition was chosen for two reasons. First, it was previously used for a different study which included surface heat flux measurements using thermocouples on the same model as in the present study. ${ }^{6,7}$ Second, it has a relatively high static pressure compared to other 
test conditions that have been previously characterized in the HET.

Table 1: Theoretical parameters for HET test condition.

\begin{tabular}{cc}
\hline \hline Condition & Air 4 \\
\hline Mach Number & 5.12 \\
Static temperature, $\mathrm{K}$ & 676 \\
Static pressure, $\mathrm{kPa}$ & 8.13 \\
Velocity, $\mathrm{m} / \mathrm{s}$ & 2664 \\
Density, $\mathrm{kg} / \mathrm{m}^{3}$ & 0.042 \\
Test time, $\mu \mathrm{s}$ & 361 \\
Unit Reynolds Number, $1 / m$ & $3.42 \mathrm{e} 6$ \\
Initial Pressures, $\mathrm{kPa}$ & \\
${ } }$ & 2500 \\
Driven Section & 6.0 \\
Expansion Section & 0.08
\end{tabular}

The model was a $63.5 \times 248 \mathrm{~mm}$, sting-mounted, flat plate with pressure sensitive paint applied to the upper surface, Figure 1. The model was also instrumented with thermocouple gauges for heat transfer measurements. Since it was not known if the PSP coating would interfere with the thermocouple junctions it was decided to leave the area over the thermocouples unpainted. (This is manifested in the PSP images as white strips on the centerline and at one spanwise location on the model.) For more information on the heat transfer instrumentation and measurements see Flaherty and Austin. ${ }^{6,7}$ A schlieren image of the laminar boundary layer developing over the front portion of the flat plate model is shown in Figure 2.

The model was constructed such that strips of protuberance elements (designed to work as vortex generators) could be interchangeably mounted $76 \mathrm{~mm}$ behind the leading edge. Two different geometries of discrete protuberance elements were used. The first was a strip of diamond shaped elements, Figure 3a. A schlieren image of the flow over the model with the diamond protuberance strip installed can be seen in Figure 4 . The second geometry was a tapered triangle design, Figure 3b. Both of these protuberance strips were based on the designs of Berry, et al. in their work on hypersonic trip development for the Hyper-X. ${ }^{8}$ The diamond trips were designed such that the height, width, and spacing $(2.5 \mathrm{~mm})$ was just over one boundary layer thicknesses for this test condition. For the triangle trips the height, tip spacing, and base width of the triangles were again $2.5 \mathrm{~mm}$.

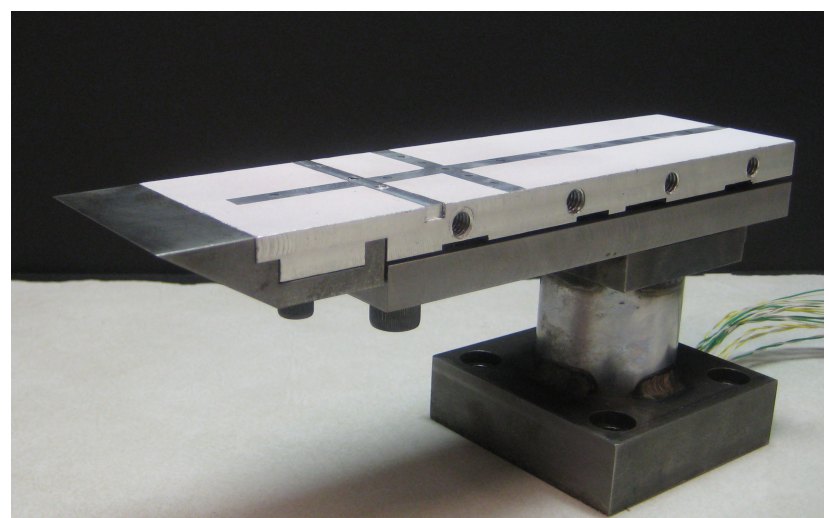

Figure 1: Flat plate model used for PSP measurements

Since the PSP has a radiative transition near $532 \mathrm{~nm}$ a frequency doubled Nd:YAG New Wave Research Gemini 15 laser was used for illumination with $90 \mathrm{~mJ} /$ pulse. An optical diffuser was used to diffuse the laser light over the top surface model. Imaging was done using a PCO 1600 CCD camera mounted with optical access through the top window of the test section. 


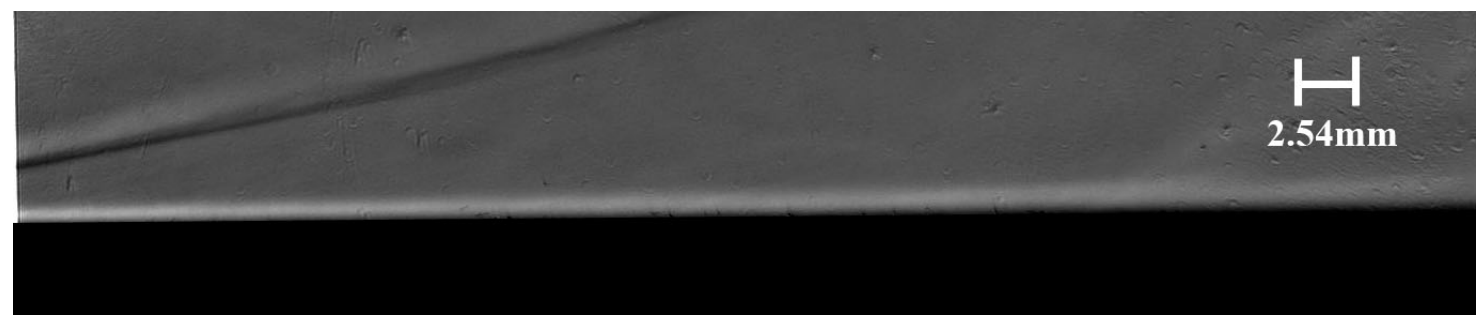

Figure 2: Schlieren visualization of laminar boundary layer development in a Mach 5.12 freestream over flat plate (flow from left to right).

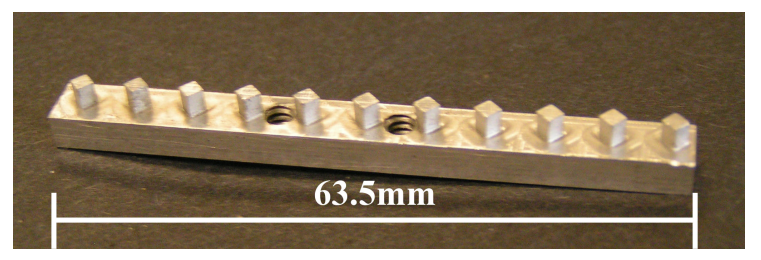

(a)

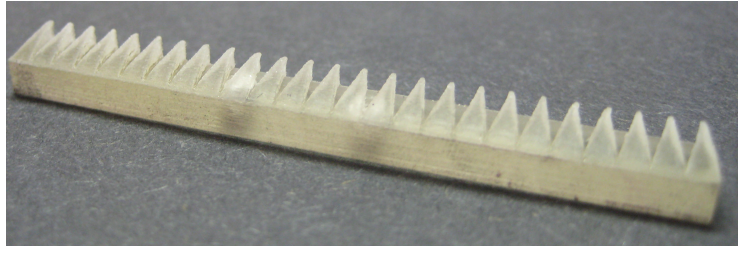

(b)

Figure 3: Two arrays of protuberances: a) diamond (pizza-box) geometry and b) tapered triangle geometry.

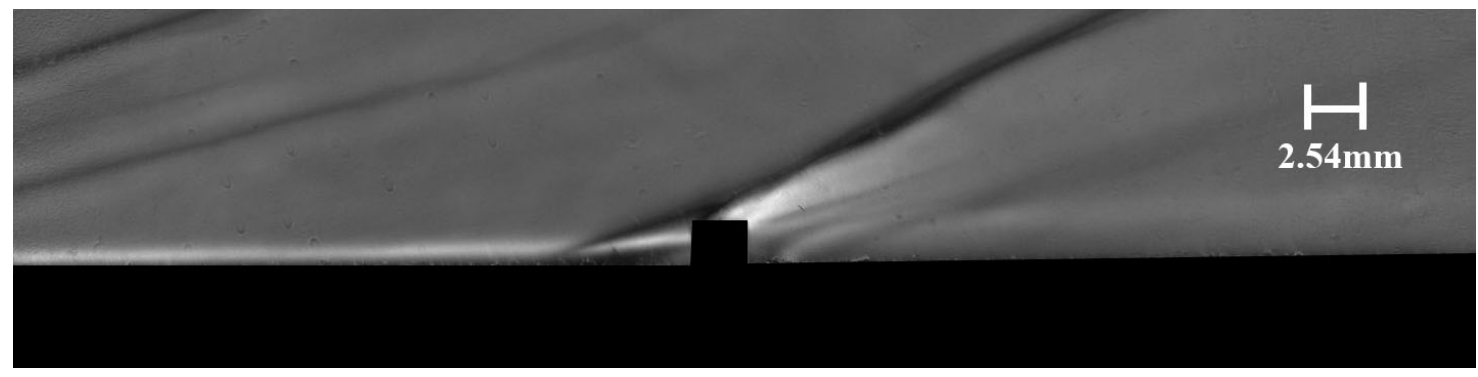

Figure 4: Schlieren image of flow over flat plate with diamond protuberance strip installed $76 \mathrm{~mm}$ downstream of the leading edge (flow from left to right). 
There are two different methods of collecting PSP data: radiative and lifetime. In the radiative method, the laser pulse occurs during the test time. The camera image acquisition is triggered simultaneously with the laser pulse, and the exposure time is long enough that a good signal level is attained. For this method the camera averages over the entire duration of the paint luminescence. The ratio of this "wind-on" image is taken with a "wind-off" image (no flow over the model) at a known pressure. This ratio should eliminate most of the effects of non-uniform lighting and paint thickness. In the lifetime method, the laser pulse again occurs during the test time, however the camera is operated in double shutter mode. The first image is taken with the specified exposure time. After the exposure time the process of reading out this image begins and, after a small delay ( $180 \mathrm{~ns})$, the second image recording begins. The limitation of this technique is that the exposure of the second image cannot be directly controlled and can vary from $22-208 \mathrm{~ms}$. During post processing, the ratio of the two images taken during the test time is taken in order to eliminate non-uniformities in the light sheet and paint. Even though test times in the HET are on the order of hundreds of microseconds, the long exposure of the second frame was not believed to be a problem since the radiative lifetime of the paint is only a few microseconds. Thus, for most of the second exposure, the camera would only be recording background. Unfortunately, when initial experiments were run in the HET with the camera in double shutter mode it was discovered that the arrival of the driver gas in the test section results in significant natural luminescence in the gas. Even though the intensity of this luminescence is much lower than the laser intensity, it is still enough to completely saturate the second image during its exposure time. This makes the second image useless. Solutions to this issue are currently under investigation, but the data presented here were all taken in the radiative mode of operation.

For comparison with the PSP data, static pressure measurements were made using Endevco Model $8530 \mathrm{C}-15$ piezoresistive pressure transducers. These gauges have a response time of $70 \mathrm{kHz}$. The gauges were calibrated in situ in the same manner as the PSP during the evacuation of the HET. The output of the gauges was filtered through a $80 \mathrm{kHz}$, low pass analogue filter in order to eliminate the effects of the resonance frequency of the gauge.

\section{Results}

PSP measurements were made over the surface of the flat plate model with no protuberance element installed, Figure 5, over the flat plate with the diamond protuberances, Figure 6 , and over the flat plate with tapered triangle protuberances, Figure 7 . With no elements installed there is very little variation in the pressure in the spanwise direction across the flat plate, indicating edge effects are not present in the region of interest. For the both the diamond and triangular strips, strong vortical structures are seen to form behind the protuberances. The vortices appear to breakdown some distance downstream of the strip. To estimate the region in which the breakdown occurs for comparative measurements, the data were processed as binary images with an intensity threshold varied to $90 \%$. The streamwise location at which a coherent vortex structure was no longer evident was recorded for each vortex as a function of the threshold. Recording the minimum and maximum values over all the vortices allows us to estimate the streamwise location and extent of the breakdown. For the diamond protuberances, vortex breakdown was recorded from 34 to $38 \mathrm{~mm}$ downstream, Figure 6. For the triangular protuberances, the vortex breakdown region was located further upstream in the un-painted area (corresponding to the thermocouple locations) between 18 and $25 \mathrm{~mm}$ behind the leading edge.

The individual vortices formed behind the triangular protuberances are much smaller in diameter than those formed by the diamond protuberance. This is to be expected as the triangle elements have a significantly different shape and would be expected to create a different vortex distribution. Spanwise distributions were taken from all three cases, Figure 8. For the baseline flat plate, there are high frequency fluctuations associated with the noise in the data. For the two strips, coherent, large scale fluctuations can be seen which are representative of the width of the vortices. The size of these vortices is approximately equal to the width of the space between the protuberance elements.

Static pressure measurements were made for the flat plate with no protuberance elements and with the diamond protuberances installed. These data, compared to the perfect gas freestream static pressure prediction, are summarized in Table 2. There is very good agreement between the Endevco gauge measurements and the perfect gas prediction. The PSP data and the predictions or Endevco data disagree by a factor of up to three. This indicates that there are issues either with the method of calibration, or with the radiative method of data collection for the PSP. Since the calibration method has been used in other facilities with 


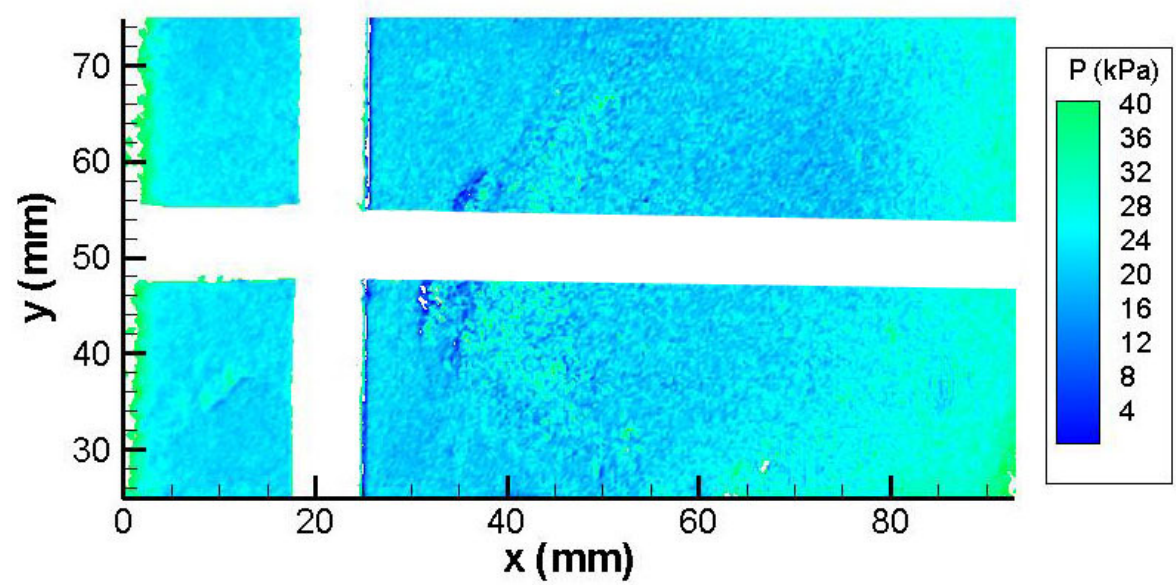

Figure 5: PSP image of flat plate with no protuberance element (white area represents unpainted area corresponding to thermocouple locations).

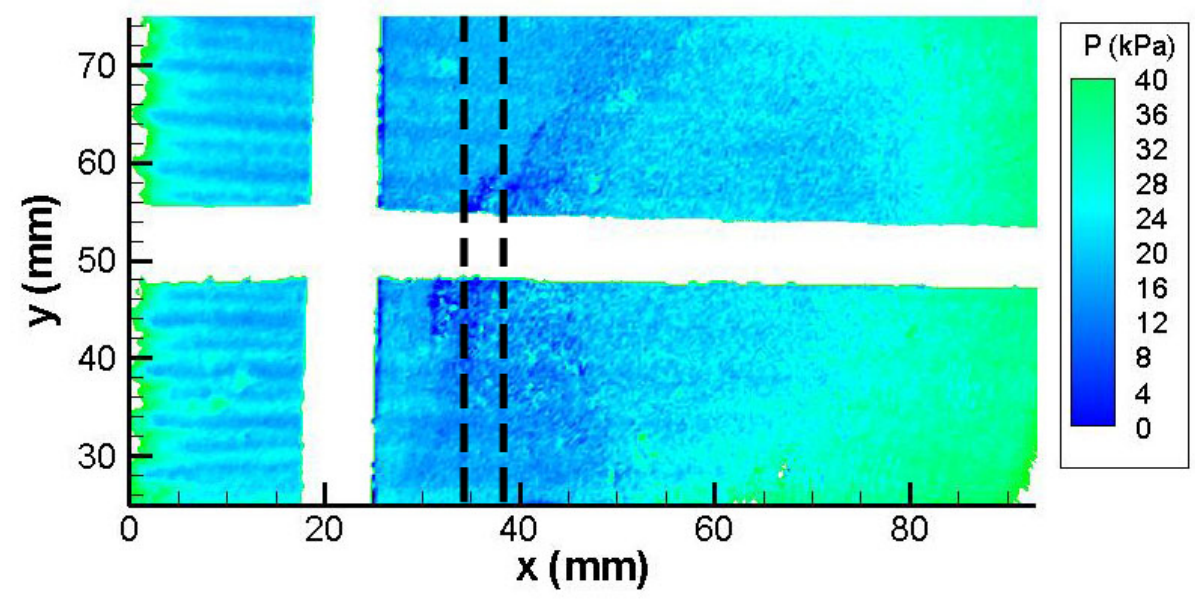

Figure 6: PSP measurements over flat plate with diamond protuberance elements (elements are spaced at $2.5 \mathrm{~mm}$ ). Dashed lines indicate region of vortex breakdown.

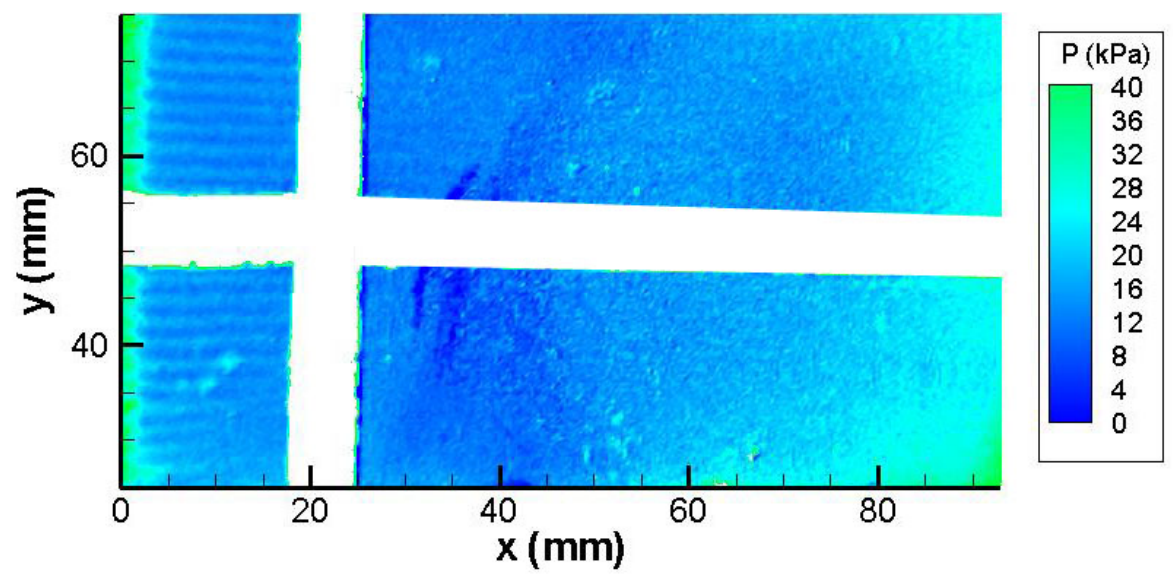

Figure 7: PSP image of flat plate with tapered triangle protuberance element (elements are spaced at $2.5 \mathrm{~mm})$. 


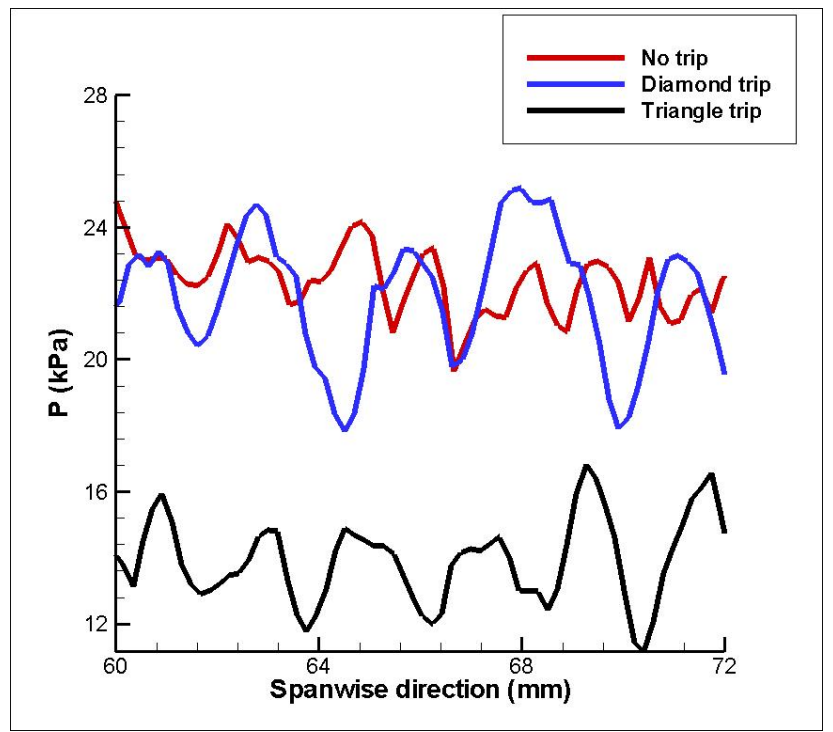

Figure 8: Spanwise pressure cross-sections over the flat plate with no protuberances, and two different protuberance geometries.

success, the use of the radiative method should first be investigated and future experiments using the lifetime method in order to obtain better accuracy for the pressure levels are planned.

Table 2: Comparison of Edevco gauge data to PSP data

\begin{tabular}{|c|c|c|c|}
\hline Configuration & Perfect Gas $(\mathrm{kPa})$ & Endevco $(\mathrm{kPa})$ & $\mathrm{PSP}(\mathrm{kPa})$ \\
\hline No protuberance & 8.13 & $8.40 \pm 0.84$ & 20.68 \\
\hline Diamond protuberance & - & $9.12 \pm 0.91$ & 18.36 \\
\hline
\end{tabular}

\section{Conclusion and Future Work}

The goal of this paper was to investigate the application of fast-responsing PtTFPP porous polymer paint in a hypervelocity flow environments. Tests were carried out in the Hypervelocity Expansion Tube in a Mach 5.12 freestream. Surface pressure data were successfully obtained over a baseline flat plate model, and also over the model with two different protuberance geometries acting as vortex generators. The response time of the paint was demonstrated to be sufficient to obtain qualitative pressure distributions and streamwise vortex visualizations over multiple model geometries in a hypersonic test environment. The vortex structure, frequency and breakdown distance could be estimated from the data. At the current time, the radiative method does not produce quantitative pressure levels in acceptable agreement with pressure tap data. Future work will concentrate on implementing the lifetime method of data collection.

\section{Acknowledgments}

This work was supported in part by the Air Force Research Laboratory through award FA8650-10-M3023 with Dr. Daniel Tejtel as Program Manager and in part by the Air Force Office of Scientific Research through award FA9550-08-1-0172 with Dr. John Schmisseur as Program Manager.

\section{References}

\footnotetext{
${ }^{1}$ Crafton, J., Forlines, A., Palluconi, S., Hsu, K.-Y., Carter, C., and Gruber, M., "Investigation of transverse jet injections in a supersonic crossflow using fast responding pressure-sensitive paint," 49th AIAA Aerospace Sciences Meeting, Orlando FL, Jan 2011.

${ }^{2}$ Asai, K., Kanda, H., Cunningham, C. T., Erausquin, R., and Sullivan, J. P., "Surface pressure measurement in a cryogenic
} 
wind tunnel by using luminescent coating," 17th International Congress on Instrumentation in Aerospace Simulation Facilities, Institute of Electrical and Electronics Engineers, Inc., Piscataway, NJ, 1997.

${ }^{3}$ Sakaue, H. and Sullivan, J. P., "Fast response time characteristics of anodized aluminum pressure sensitive paint," 38th Aerospace Sciences Meeting and Exhibit, AIAA 2000-506, Reno, NV, Jan 2000.

${ }^{4}$ Gregory, J. W., Asai, K., Kameda, M., Liu, T., and Sullivan, J. P., "A review of pressure-sensitive paint for high-speed and unsteady aerodynamics," Proceedings of the Institutaion of Mechanical Engineers, Part G: Journal of Aerospace, Vol. 222, No. 2, 2008, pp. 249-290.

${ }^{5}$ Dufrene, A., Sharma, M., and Austin, J., "Design and characterization of a hypervelocity expansion tube facility," Journal of Propulsion and Power, Vol. 23, No. 6, 2007, pp. 1185-1193.

${ }^{6}$ Flaherty, W. and Austin, J. M., "Comparative surface heat transfer measurements in hypervelocity flow," 48th AIAA Aerospace Sciences Meeting, AIAA 2010-671, Orlando FL, Jan 2010.

${ }^{7}$ Flaherty, W. and Austin, J. M., "Effect of concave wall geometry on heat transfer in hypersonic boundary layers," 40th Fluid Dynamics Conference and Exhibit, AIAA 2010-4986, Chicago IL, June 2010.

${ }^{8}$ Berry, S. A., Auslender, A. H., Dilley, A. D., and Calleja, J. F., "Hypersonic boundary-layer trip development for Hyper-X," Journal of Spacecraft and Rockets, Vol. 38, No. 6, 2001, pp. 853-864. 